\title{
Sensibilidade da permeabilidade e da incompressibilidade de rochas carbonáticas à pressão confinante
}

\author{
Freire, L.A ${ }^{1}$; Soares ${ }^{*}$, J.A ${ }^{1}$, Sousa,W.B ${ }^{1}$, Cavalcanti, B.M ${ }^{1}$
}

${ }^{1}$ Universidade Federal de Campina Grande

Copyright 2014, SBGf - Sociedade Brasileira de Geofísica

Este texto foi preparado para a apresentação no VI Simpósio Brasileiro de Geofísica, Porto Alegre, 14 a 16 de outubro de 2014. Seu conteúdo foi revisado pelo Comitê Técnico do VI SimBGf, mas não necessariamente representa a opinião da SBGf ou de seus associados. É proibida a reprodução total ou parcial deste material para propósitos comerciais sem prévia autorização da SBGf.

\section{Resumo}

O presente trabalho investiga os efeitos do aumento da pressão confinante sobre a permeabilidade e a incompressibilidade de rochas carbonáticas. As amostras analisadas são provenientes das bacias do Araripe, Sergipe-Alagoas e Potiguar. Foram realizados ensaios de permeabilidade sob cinco níveis de pressão confinante e ensaios de velocidades elásticas sob pressões confinantes entre $5 \mathrm{MPa}$ e $40 \mathrm{MPa}$. Com as velocidades elásticas e a densidade a incompressibilidade dinâmica foi calculada. Verificou-se que a permeabilidade tende a diminuir com o aumento da pressão confinante, enquanto que a incompressibilidade tende a aumentar. Os resultados mostram que não há uma relação simples entre as variações da permeabilidade e da incompressibilidade com 0 aumento da pressão confinante, sendo discutidas outras variáveis que afetam esta relação. O tipo de porosidade predominante é uma destas variáveis.

\section{Aspectos geológicos das amostras estudadas}

A Bacia do Araripe apresenta uma sequência predominantemente mesozóica. De acordo com Assine (1992), a bacia apresenta quatro sequências estratigráficas limitadas por discordâncias regionais. A sequência Aptiano-Albiana é composta pelas Formações Barbalha, Santana e Araripina. A Formação Santana, da qual amostras são analisadas neste trabalho, tem origem marinha/lacustre, apresentando uma sequência sedimentar estratificada constituída por gipsitas, calcários, siltitos argilosos, margas e folhelhos betuminosos.

A Bacia Potiguar, formada no Eojurássico, apresenta rochas organizadas em três grupos: Areia Branca, Apodi e Agulha (ARARIPE \& FEIJÓ, 1994). No grupo Apodi se encontra a Formação Jandaíra, da qual foram extraídas amostras analisadas neste trabalho. Esta formação é constituída por rochas carbonáticas, apresentando como litotipos os calcarenitos bioclásticos, às vezes associados com algas verdes, e calcilutitos com marcas de raízes e gretas de contração, com cores variando de cinza claro a amarelo.

Segundo Schaller (1969), a Formação Riachuelo (Bacia Sergipe-Alagoas) engloba um complexo clásticocarbonático, onde observam-se três membros, dentre os quais o membro Maruim, constituído por calcarenitos e calcirruditos de cor creme e dolomitos de coloração de creme a castanho. Neste trabalho são investigadas amostras deste membro.

\section{Preparo de amostras}

Inicialmente, as rochas coletadas nos afloramentos das Formações Riachuelo (Sergipe), Jandaíra (Potiguar) e Crato (Araripe), passaram por um processo de serragem no laboratório de preparo de amostras. A serragem tem o intuito de facilitar a preparação dos plugues cilíndricos de rochas, que são feitos na plugadeira de bancada, apresentando uma polegada e meia de diâmetro e altura em torno de $5 \mathrm{~cm}$. Em seguida ocorre o desbaste, realizado na retificadora de plugues. Sua finalidade é garantir faces planas e paralelas, necessárias aos ensaios de velocidade de onda. A Figura 1 mostra, respectivamente, os equipamentos utilizados na preparação de amostras.

Após esta etapa as amostras foram levadas ao laboratório de Petrofísica da UFCG, onde foram submetidas a uma secagem na estufa por um período de 24 horas em uma temperatura de $80^{\circ} \mathrm{C}$, pesagem em balança eletrônica de precisão semi-analítica e medição de suas dimensões com paquímetro digital. A Figura 2 apresenta os equipamentos utilizados. A Figura 3 mostra as amostras obtidas.

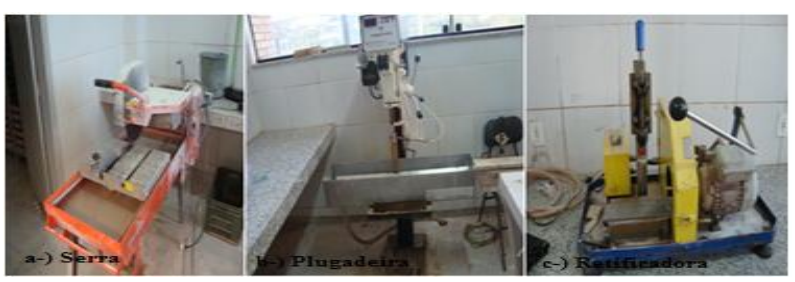

Figura 1 - Equipamentos para preparação de plugues.

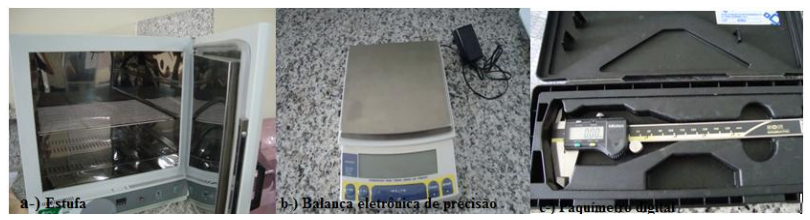

Figura 2 - Equipamentos utilizados após o preparo dos plugues.

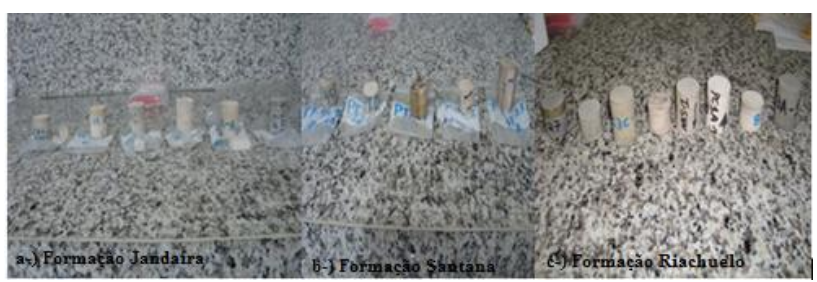

Figura 3 - Plugues extraídos de amostras de rochas carbonáticas. 
O Quadro 1 lista as amostras submetidas a ensaios petrofísicos com suas respectivas formações.

Quadro 1 - Identificação das amostras ensaiadas com suas respectivas formações e bacias.

\begin{tabular}{|l|l|}
\hline \multicolumn{1}{|c|}{ Amostra } & \multicolumn{1}{c|}{ Formação (Bacia) } \\
\hline PC3A_1 & Riachuelo (Sergipe) \\
\hline PC3C_23_V & Riachuelo (Sergipe) \\
\hline NAS_1 & Riachuelo (Sergipe) \\
\hline PC1A_1_1 & Riachuelo (Sergipe) \\
\hline PC3C_27 & Riachuelo (Sergipe) \\
\hline NAS_8 & Riachuelo (Sergipe) \\
\hline PC2J_2B & Riachuelo (Sergipe) \\
\hline PC3C_14 & Riachuelo (Sergipe) \\
\hline PT11_H1 & Santana (Araripe) \\
\hline PT9F_H4 & Santana (Araripe) \\
\hline PT09_16M) & Santana (Araripe) \\
\hline PT45_4_(10M)_1 & Santana (Araripe) \\
\hline PT_09_1(12M) & Santana (Araripe) \\
\hline CAL_RONC_V & Jandaíra (Potiguar) \\
\hline AR_OBL_V3 & Jandaíra (Potiguar) \\
\hline HPC_H1 & Jandaíra (Potiguar) \\
\hline ROSÁRIO_H & Jandaíra (Potiguar) \\
\hline
\end{tabular}

\section{Ensaios de Permeabilidade}

As análises de permeabilidade foram realizadas com o auxílio de um permoporosímetro a gás em câmara de compressão (UltraPoroPerm $500^{\circledR}$ ), modelo da Corelab, visualizado na Figura 4. O equipamento é uma combinação de um instrumento de medição de permeabilidade, porosidade e uma câmara simples de compressão hidrostática (coreholder). Na parte interna do coreholder, existe um cilindro de borracha, no qual a amostra é colocada, limitada por dois êmbolos de aço móveis e adaptáveis ao comprimento da amostra. Há uma bomba hidráulica conectada ao coreholder que é utilizada para impor pressão confinante à amostra de rocha.

O permeâmetro a gás trabalha obedecendo a lei de Darcy, de modo que, para calcular a permeabilidade, utiliza-se da seguinte expressão:

$$
\mathrm{K}=\frac{2000 \mathrm{P}_{1} \mu \mathrm{Q}_{1} \mathrm{~L}}{\mathrm{~A}\left(\mathrm{P}_{1}^{2}-\mathrm{P}_{2}^{2}\right)}
$$

onde,

$\mathrm{K}=$ permeabilidade absoluta $(\mathrm{mD})$

$\mu=$ viscosidade do nitrogênio $(\mathrm{CP})$

$\mathrm{Q}_{1}=$ taxa de fluxo de gás $\left(\mathrm{cm}^{3} / \mathrm{s}\right)$

$\mathrm{A}$ = área da seção transversal da amostra

$\mathrm{P}_{1}=$ pressão de injeção do gás (atm)

$\mathrm{P}_{2}=$ pressão de saída do gás (atm)

$\mathrm{L}=$ comprimento da amostra $(\mathrm{cm})$

Neste trabalho, foram realizados testes com pressões confinantes de 1000, 2000, 3000, 4000 e 5000 psi, com o intuito de verificar o efeito do aumento da pressão confinante sobre a permeabilidade.

\section{Ensaios elastodinâmicos}

As análises elastodinâmicas medem as velocidades de propagação das ondas $\mathrm{P}, \mathrm{S}_{1}$ e $\mathrm{S}_{2}$. Estes ensaios foram realizados no equipamento Autolab500 ${ }^{\circledR}$, fabricado pela New England Resarch, e mostrado na Figura 5. O processo consiste na medição do tempo de trânsito da transmissão direta de ondas elásticas através do comprimento axial dos plugues de rocha.

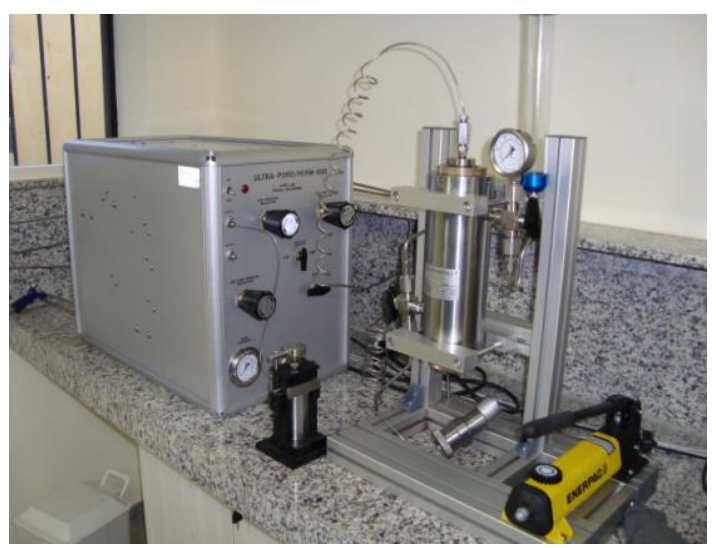

Figura 4 - Permoporosímetro a gás.

Os ensaios foram realizados com as amostras secas, e com pressão de poros e temperatura nas condições normais do ambiente. A pressão confinante inicial foi estabelecida em $40 \mathrm{MPa}$ e, após um processo de estabilização que dura em torno de 25 minutos, as medições seguintes são realizadas em ordem decrescente de $5 \mathrm{MPa}$, com tempos de estabilização entre 2 a 3 minutos. Este processo é continuado até atingir o valor mínimo da pressão confinante de $5 \mathrm{MPa}$. Todas as ondas são visualizadas no osciloscópio do equipamento. Em seguida ocorre a captura da onda pelo software de aquisição e controle. Por fim, o software realiza o processamento das ondas e gera um relatório em formato pdf contendo resultados como módulos de Young, coeficientes de Poisson e velocidades elásticas.

A velocidade de cada onda elástica é determinada pela razão entre o comprimento da amostra e o tempo de propagação daquela onda. Com os módulos de Young e os coeficientes de Poisson, determinam-se os valores de incompressibilidade das amostras para todos os níveis de pressão confinante do ensaio, de acordo com as relações entre os módulos elásticos, conforme descrito em Sheriff (1991).

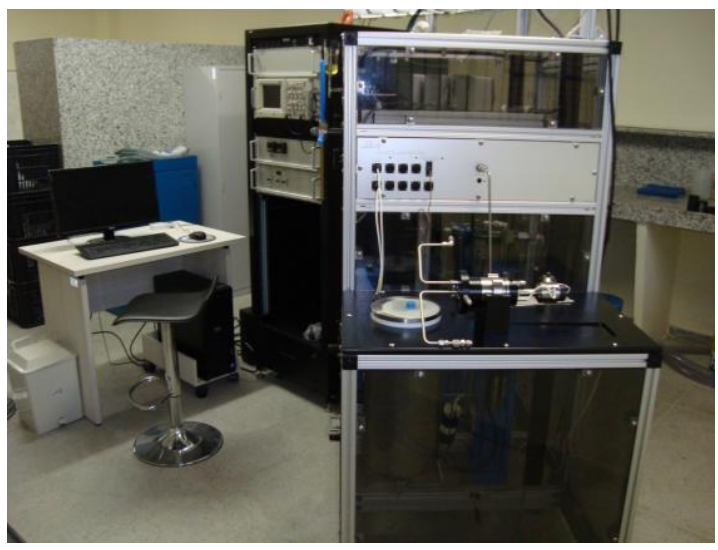

Figura 5 - Câmara de medição de velocidades elásticas. 


\section{Resultados e Discussões}

A Figura 6 mostra o resultado do aumento da pressão confinante na permeabilidade. Percebe-se que basicamente as amostras apresentaram um comportamento semelhante, tendo diminuído as suas permeabilidades com o aumento da pressão confinante. Isto acontece devido ao aumento da compressão da amostra dentro do coreholder, reduzindo os espaços utilizados para o fluxo do gás. No entanto, as permeabilidades medidas se encontram dentro de um amplo intervalo de valores, variando cinco ordens de grandeza, desde $10^{-4} \mathrm{mD}$ até $10^{+1} \mathrm{mD}$. Vê-se nesta figura que há um grupo de amostras de alta permeabilidade (CAL_RONC_V, NAS_1, ROSARIO_H, etc.). A maioria das amostras se encontra em um intervalo de permeabilidades medianas (em torno de $10^{-1} \mathrm{mD}$ ), enquanto que outras, em menor número, apresentam valores de permeabilidade igual ou menor do que $10^{-2}$ $\mathrm{mD}$.

Pode-se observar da Figura 6 que algumas amostras apresentam variações (reduções) mais fortes da permeabilidade com o aumento da pressão confinante, enquanto que em outras amostras essa redução da permeabilidade com o aumento da pressão confinante é menor. Isto acontece de forma independente do nível de permeabilidade.

A Figura 7 apresenta as incompressibilidades calculadas a partir das velocidades elásticas. Neste gráfico é possível visualizar que o aumento da pressão confinante provoca aumento da incompressibilidade das amostras. Em outras palavras, isto expressa 0 aumento de resistência mecânica que a amostra apresenta para se deformar (compactar) quando submetida a pressões crescentes de confinamento.

Percebe-se que as incompressibilidades se encontram na faixa de valores entre $10 \mathrm{GPa}$ e $50 \mathrm{GPa}$. A maior parte das amostras apresenta incompressibilidade acima de 30 GPa (LAJ_SOL_V, ROSARIO_H, PT9F_H4, etc.), apresentando portanto uma alta incompressibilidade. Uma outra parte das amostras, em menor número, encontra-se em uma faixa variando de $20 \mathrm{GPa}$ até 30 $\mathrm{GPa}$ (incompressibilidades medianas), e por fim, ocorre algumas amostras abaixo de $20 \mathrm{GPa}$ (baixa incompressibilidade).

Independente do nível de incompressibilidade, se observa que algumas amostras apresentam alta variação da incompressibilidade com 0 aumento da pressão confinante, enquanto que em outras amostras essa variação é bem menor.

A equação (2) foi utilizada como uma função de ajuste para os valores de permeabilidade medidos em função da pressão confinante (ZIMMERMAN, 1991).

$$
\log (\mathrm{K})=\log \left(\mathrm{K}_{\mathrm{HS}}\right)\left[1-\left(\frac{\Delta}{1+\Delta}\right) \exp \left(\frac{-\mathrm{P}_{\mathrm{CONF}}}{\mathrm{P}_{\mathrm{C}}}\right)\right]
$$

Onde:

$\log (K)=$ logaritmo da permeabilidade;

$\log \left(K_{H S}\right)=$ logaritmo da permeabilidade sob alta pressão confinante;

$\Delta=$ máxima variação no valor de permeabilidade;

$\mathrm{P}_{\mathrm{CONF}}=$ pressão confinante;
$P_{C}=$ Pressão crítica. Corresponde ao ponto de inflexão da curva de permeabilidade versus pressão confinante. Quanto menor $\mathrm{P}_{\mathrm{c}}$, maior a concavidade da curva.

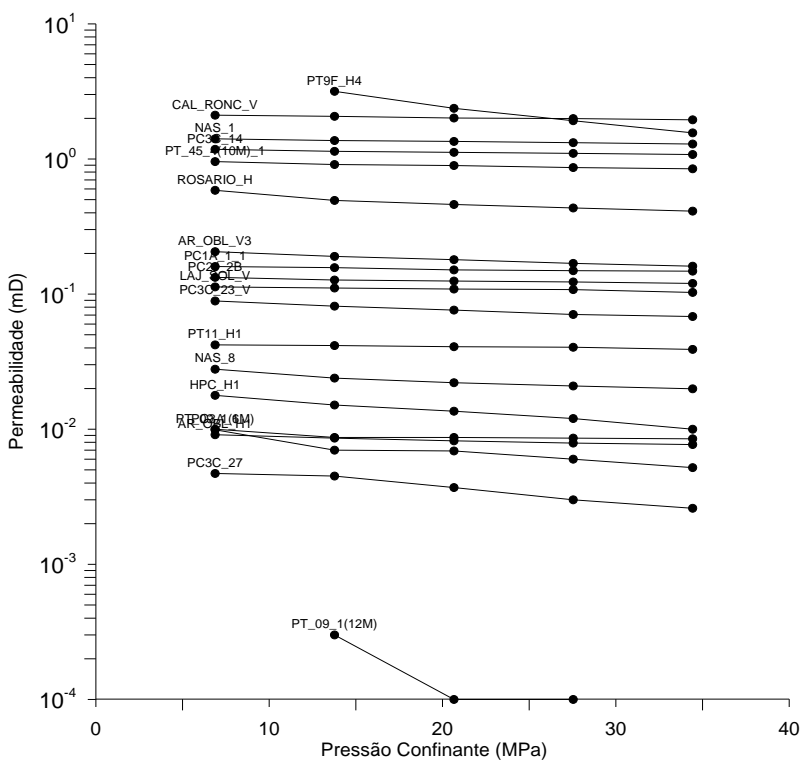

Figura 6 - Efeito do aumento da pressão confinante na permeabilidade.

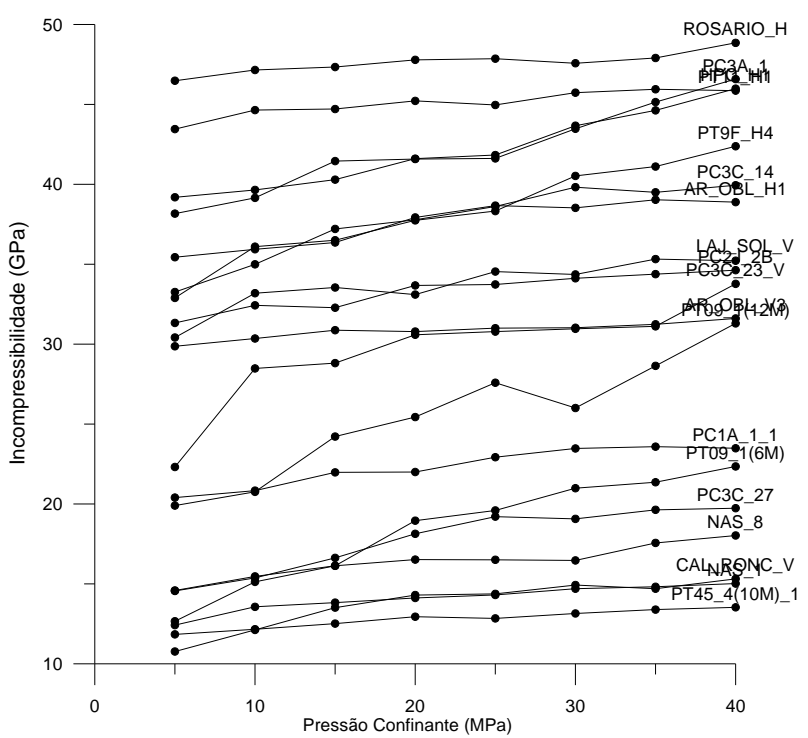

Figura 7 - Incompressibilidade medida com o aumento da pressão confinante.

Para o ajuste dos valores de incompressibilidade com a pressão confinante se utiliza basicamente a mesma equação (2), mas sem a utilização da função logarítmica.

A Figura 8 apresenta os valores do logaritmo da permeabilidade sob alta pressão confinante (K $\mathrm{K}_{\mathrm{HS}}$-Perm) versus a incompressibilidade também sob alta pressão confinante (KHS-GPa). No eixo das ordenadas vê-se que as permeabilidades das amostras se encontram no intervalo de ordens de grandeza que vão de -4 até +1 , enquanto que o eixo das abcissas indica um intervalo de incompressibilidades que vai de 10 a $50 \mathrm{GPa}$. 
Pode-se notar que não há uma relação clara entre as variáveis confrontadas nesta figura. As razões para isto são variadas. Por exemplo, o comportamento esperado para um material poroso e homogêneo é que amostras de elevada porosidade sejam mais permeáveis e também mais compressíveis. No entanto, se os poros forem muito pequenos, uma amostra com alta microporosidade apresenta baixa permeabilidade, embora possa ser bastante compressível. Também se espera que um material de baixa porosidade seja mais incompressível e de baixa permeabilidade. Isto é verdadeiro se a porosidade for do tipo intergranular.

Se a porosidade for do tipo vugular com poros interconectados, mesmo uma amostra de relativamente baixa porosidade pode apresentar uma alta permeabilidade, embora tenda a apresentar também uma elevada incompressibilidade. Já se os vugs apresentarem um baixo índice de conectividade, embora a incompressibilidade continue sendo elevada, a permeabilidade será muito baixa.

As relações gerais acima discutidas podem explicar a grande dispersão dos dados apresentados na Figura 8. Por exemplo, as amostras CAL_RONC_V, NAS 1 e PT45_4(10M)_1 apresentam alta permeabilidade e baixa incompressibilidade. Como se pode ver da Figura 9, estas amostras apresentam alta porosidade, e o fato de serem bastante compressíveis indica que o tipo de porosidade predominante é do tipo intergranular.

Já as amostras PC3C_14, PT9F_H4, e ROSARIO_H apresentam alta permeabilidade como também alta incompressibilidade, apesar de apresentarem porosidades médias a baixas. Este comportamento é compatível com amostras cuja porosidade predominante é do tipo vugular com poros interconectados.

Há também amostras que apresentam baixa porosidade e baixa permeabilidade, o que acontece tanto para amostras de alta quanto de baixa incompressibilidade. Neste caso a incompressibilidade da amostra também é controlada pelo tipo de porosidade. Quando essa porosidade é do tipo intergranular, a incompressibilidade tende a ser baixa e quando ela é do tipo vugular a incompressibilidade tende a ser alta, embora neste caso essa porosidade vugular seja predominantemente não interconectada. Por exemplo, as amostras HPC_H1 e PC3A_1 apresentam baixa permeabilidade, baixa porosidade e alta incompressibilidade, o que indica que a porosidade delas é provavelmente do tipo vugular com poros desconectados. Já a amostra PC3C_27 apresenta baixa permeabilidade, baixa porosidade e baixa incompressibilidade, indicando que a porosidade é predominantemente do tipo intergranular.

A Figura 9 apresenta o gráfico da porosidade versus a densidade de grãos. A densidade de grãos de rochas carbonáticas calcárias é em torno de $2,71 \mathrm{~g} / \mathrm{cm}^{3}$, entretanto é possível notar que a maior parte das amostras (PT_45_4(10M)_1, NAS_8, NAS_1, PT_09_1(12M), etc.) apresentam densidade de grãos inferior a 2,71 $\mathrm{g} / \mathrm{cm}^{3}$, o que significa dizer que estas amostras contém matéria orgânica, areia ou argila na sua composição. As amostras PC3C_27, CAL_RONC_V, PC3C_14, LAJ_SOL_V e PC3C_23_V apresentam densidade de grãos superior a $2,71 \mathrm{~g} / \mathrm{cm}^{3}$, o que indica que sofreram um processo de dolomitização, total ou parcial. A PT11_H1 trata-se de um dolomito puro, pois tem uma densidade de grãos acima de $2,8 \mathrm{~g} / \mathrm{cm}^{3}$, enquanto que as demais, por apresentarem densidades de grãos abaixo de $2,8 \mathrm{~g} / \mathrm{cm}^{3}$, foram apenas parcialmente dolomitizadas.

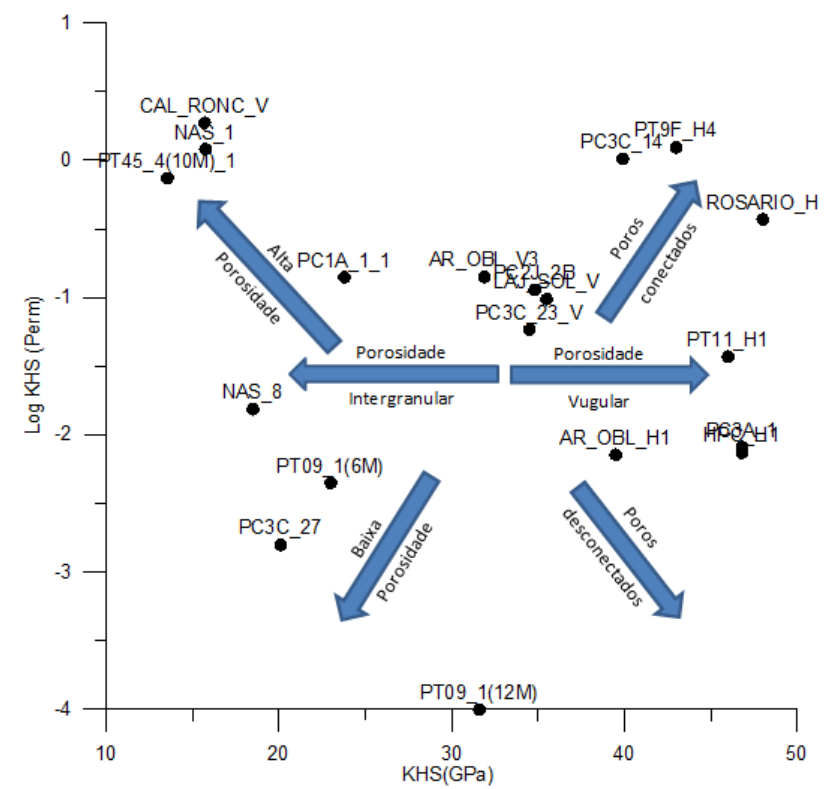

Figura 8 - Relação entre o logaritmo da permeabilidade, e a incompressibilidade, ambos sob alta pressão confinante.

A Figura 10 apresenta o gráfico da pressão crítica do logaritmo da permeabilidade versus a pressão crítica da incompressibilidade. A pressão crítica é a variável que controla o grau de concavidade da curva de ajuste entre a variável medida e a pressão confinante. Para a incompressibilidade, o intervalo de valores estimados varia entre 7,6 até 20,6 MPa. Já a pressão crítica estimada para o logaritmo da permeabilidade se encontra no intervalo entre 5 e $43 \mathrm{MPa}$. Portanto, a pressão crítica do logaritmo da permeabilidade apresentou uma maior variação de valores, indicando curvas de ajuste com uma maior distribuição de graus de concavidade, pois a concavidade da curva de ajuste é inversamente proporcional à pressão crítica. Ou seja, baixo valor de Pc indica uma curva de ajuste de elevada concavidade, enquanto que um alto valor de Pc indica uma curva mais retilínea.

A Figura 11 apresenta o gráfico da máxima variação do logaritmo da permeabilidade versus a máxima variação da incompressibilidade. Desta forma, uma máxima variação com valor alto, em módulo, indica que a variável analisada sofre uma maior variação dos seus valores entre a menor e a maior pressão confinante. Sempre que a permeabilidade de uma amostra for maior que a unidade, em miliDarcy, o delta do seu logaritmo será negativo, e o delta será positivo quando a permeabilidade for menor que a unidade. De modo geral, o que se observa nesta figura é que não há uma relação clara entre as variáveis analisadas. 


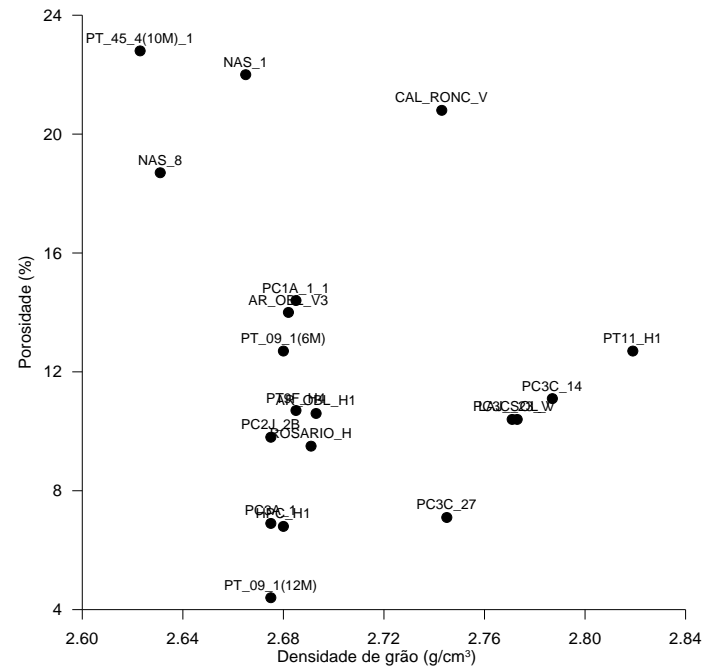

Figura 9 - Porosidade versus densidade de grãos.

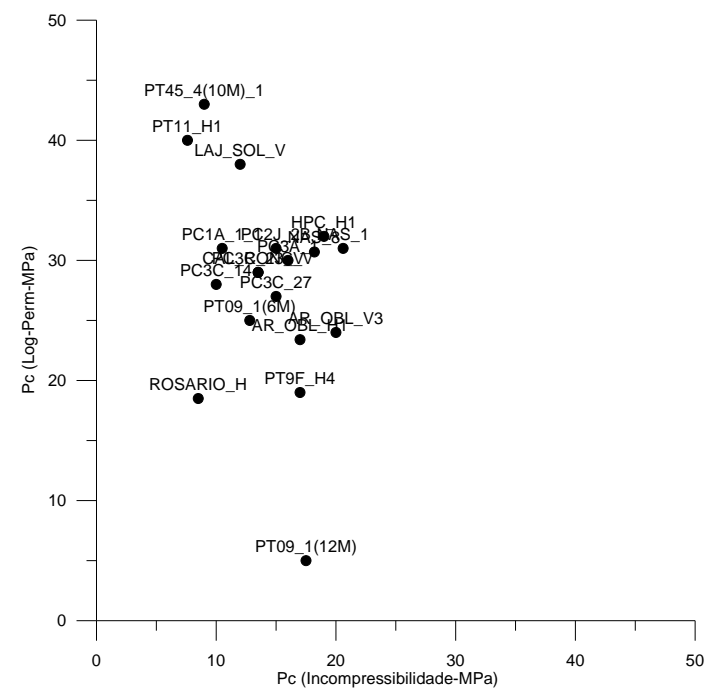

Figura10 - Pressão crítica do logaritmo da permeabilidade versus a pressão crítica da incompressibilidade.

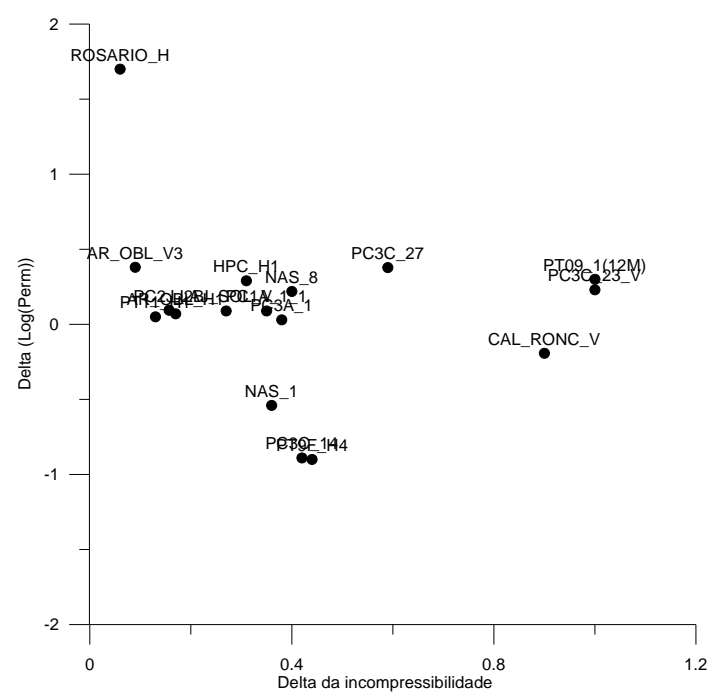

Figura 11 - Máxima variação do logaritmo da permeabilidade versus a máxima variação da incompressibilidade.

\section{Conclusões}

Os resultados apresentados mostram que a permeabilidade diminui enquanto a incompressibilidade aumenta com o aumento da pressão confinante. Nota-se que, independente do nível de permeabilidade, algumas amostram apresentam variações mais fortes de permeabilidade e de incompressibilidade com o aumento da pressão confinante do que outras.

Não há uma relação simples entre o aumento da incompressibilidade e a redução da permeabilidade das amostras como decorrência do aumento da pressão confinante. Outras variáveis, além da pressão confinante, afetam esta relação. $O$ tipo de porosidade predominante é uma destas variáveis.

Se a porosidade da amostra for predominantemente do tipo intergranular, a incompressibilidade sob alta pressão confinante será baixa; enquanto que, se a porosidade for majoritariamente vugular, essa incompressibilidade será alta. Por outro lado, uma porosidade intergranular alta resulta em uma alta permeabilidade sob confinamento elevado, enquanto que uma porosidade intergranular baixa está associada a baixos valores de permeabilidade sob altas pressões confinantes. Já no caso da porosidade vugular, a permeabilidade sob pressão confinante máxima será alta se os poros estiverem interconectados e será baixa no caso de poros isolados.

Os efeitos de outras variáveis precisam ser ainda avaliados: microporosidade, razão de aspecto e arranjo de grãos, presença de descontinuidades (fissuras e planos de fraqueza), processos diagenéticos, anisotropia elástica, entre outras.

A medição da densidade de grãos das amostras de rochas carbonáticas se mostrou como uma importante ferramenta para a identificação da composição mineral das amostras. Amostras carbonáticas com densidade de grãos inferior a $2,71 \mathrm{~g} / \mathrm{cm}^{3}$ provavelmente estão afetadas pela presença de matéria orgânica, areia ou argila. Valores superiores a $2,71 \mathrm{~g} / \mathrm{cm}^{3}$ estão associados a processo de dolomitização, que pode ser parcial ou total.

\section{Referências}

ASSINE, M. L. Análise Estratigráfica da Bacia do Araripe, Nordeste do Brasil. Revista Brasileira de Geociências, São Paulo, v.22, n. 3, p. 289-300, 1992.

SCHALLER, H. Revisão estratigráfica da Bacia de Sergipe/Alagoas. Boletim de Geociências da Petrobras, Rio de Janeiro, v. 12, n.1, p. 21-86, 1969.

ARARIPE, P. T; FEIJÓ, F. J. Bacia Potiguar. Boletim de Geociências da Petrobras, Rio de Janeiro, v. 8, n. 2, p127-141, jan/mar. 1994.

ZIMMERMAN, R.W. (1991). Compressibility of sandstones. Elsevier Science Pub.

SOARES, J. A. Propriedades Físicas das Rochas. Boletim da SBGF. Rio de Janeiro: Sociedade Brasileira de Geofísica, n.1, 2011.

SHERIFF, R.E. (1991). Encyclopedic dictionary of exploration geophysics. Third Edition. Society of Exploration Geophysics. 